\title{
A NOTE ON THE BOUNDEDNESS OF BERGMAN-TYPE OPERATORS ON MIXED NORM SPACES
}

\author{
ZENGJIAN LOU
}

(Received 29 June 2005; revised 19 October 2005)

Communicated by P. C. Fenton

\begin{abstract}
We prove the boundedness of Bergman-type operators on mixed norm spaces $L^{p . q}(\varphi)$ for $0<q<1$ and $0<p \leq \infty$ of functions on the unit ball of $\mathbb{C}^{n}$ with an application to Gleason's problem.

2000 Mathematics subject classification: primary 47B38; secondary 32A30, 46E15.

Keywords and phrases: Bergman-type operators, mixed norm spaces, boundedness.
\end{abstract}

\section{Introduction}

Let $B$ denote the open unit ball of the complex vector space $\mathbb{C}^{n}, v$ be the Lebesgue measure on $\mathbb{C}^{n}$ normalized so that $v(B)=1$, and let $\sigma$ be the surface measure on the boundary $\partial B$ of $B$. A positive continuous function $\varphi$ on $[0,1)$ is normal (see [4]) if there exist positive numbers $a<b$ and $0 \leq r_{0}<1$ such that:

(1) $\frac{\varphi(r)}{(1-r)^{a}}$ is nonincreasing for $r_{0} \leq r<1$ and $\lim _{r \rightarrow 1^{-}} \frac{\varphi(r)}{(1-r)^{a}}=0$;

(2) $\frac{\varphi(r)}{(1-r)^{b}}$ is nondecreasing for $r_{0} \leq r<1$ and $\lim _{r \rightarrow 1^{-}} \frac{\varphi(r)}{(1-r)^{b}}=\infty$.

The $a, b$ in the definition are not uniquely related to $\varphi$. Let $a_{\varphi}$ denote the superemum of all possible $a$ 's and $b_{\varphi}$ denote the infimum of all possible $b$ 's. We say that $a_{\varphi}$ and $b_{\varphi}$ are characteristic exponents of $\varphi$.

The author was supported by NNSF of China (Grant No. 10371069), NSF of Guangdong Province (Grant No. 06105648 and Grant No. 05008289) and SRF for ROCS, SEM.

(C) 2007 Australian Mathematical Society 1446-7887/07 \$A2.00+0.00 
For a positive continuous function $\varphi$ on $[0,1)$ and $0<p, q \leq \infty$, let $L^{p, q}(\varphi)$ denote the usual space of measurable functions $f$ on $B$ with $\|f\|_{p, q, \varphi}<\infty$, where

$$
\|f\|_{p, q, \varphi}= \begin{cases}\left(\int_{0}^{1} r^{2 n-1}(1-r)^{-1} \varphi^{p}(r) M_{q}^{p}(r, f) d r\right)^{1 / p}, & 0<p<\infty, \\ \sup _{0<r<1} \varphi(r) M_{q}(r, f), & p=\infty,\end{cases}
$$

and

$$
M_{q}(r, f)= \begin{cases}\left(\int_{\partial B}|f(r \zeta)|^{q} d \sigma(\zeta)\right)^{1 / q}, & 0<q<\infty \\ \sup _{\zeta \in \partial B}|f(r \zeta)|, & q=\infty\end{cases}
$$

Suppose $s \in \mathbb{R}$ and $t>0$ (here and afterward in this note). The Bergman-type operator $P_{s, t}$ on $L^{p \cdot q}(\varphi)$ is given by

$$
P_{s, t} f(z)=c_{n, t}\left(1-|z|^{2}\right)^{s} \int_{B} \frac{\left(1-|w|^{2}\right)^{t-1} f(w)}{(1-\langle z, w\rangle)^{n+t+s}} d v(w), \quad f \in L^{p . q}(\varphi), z \in B
$$

where $c_{n, t}=\Gamma(n+t) /(\Gamma(t) \Gamma(n+1))$ and $\langle z, w\rangle=\sum_{i=1}^{n} z_{i} \bar{w}_{i}$ for $z=\left(z_{1}, \ldots, z_{n}\right)$, $w=\left(w_{1}, \ldots, w_{n}\right)$.

The boundedness of Bergman-type operators $P_{s . t}$ on mixed norm spaces $L^{p, q}(\varphi)$ has been studied extensively; see, for example, $[3,4]$ and references cited therein. Ren and Shi showed in [4], that if $t>b>a>-s$, then $P_{s, t}$ is a bounded operator on $L^{p, q}(\varphi)$ for $1 \leq p, q \leq \infty$. Liu proved the case for $0<p<1,1<q<\infty$ in [3]. The only unsolved case is for $0<q<1$. Since both the results in [4] and in [3] rely on Hölder's inequality for $1 \leq q<\infty$ (see [4, Lemma 2.1] and [3, Lemma 3]), the idea used there cannot deal with the case $0<q<1$. In this note, by using an inequality due to Beatrous and Burbea [1], we prove that $P_{s, t}$ is bounded on $L^{p, q}(\varphi)$ for $0<q<1$ and $0<p \leq \infty$.

THEOREM 1.1. Let $\varphi$ be a normal function with characteristic exponents $a_{\varphi}$ and $b_{\varphi}$. For $0<q<1$ and $0<p \leq \infty$, if $t>n(1 / q-1)+b_{\varphi}$ and $s>-a_{\varphi}$, then $P_{s, t}$ is a bounded operator on $L^{p, q}(\varphi)$.

In this note, $C$ denotes a constant independent of functions. Such a $C$ may differ at different occurrences.

\section{Proof of Theorem 1.1}

To prove Theorem 1.1 we need the following lemmas. 
LEMMA 2.1. Suppose $f:[0,1) \rightarrow[0, \infty)$ is increasing, $\alpha, \beta>0,0 \leq \rho<1$ and $0<p \leq 1$. Then there exists a constant $C$ such that

$$
\left(\int_{0}^{1} \frac{(1-r)^{\alpha-1}}{(1-r \rho)^{\beta}} f(r) d r\right)^{p} \leq C \int_{0}^{1} \frac{(1-r)^{p \alpha-1}}{(1-r \rho)^{p \beta}} f(r)^{p} d r .
$$

The proof of Lemma 2.1 follows ideas of Hardy and Littlewood [2]. For the completeness of the paper we prove it below.

PROOF. For $0 \leq \rho<1$ and $\beta \geq 0$, the function $f(r) /(1-\rho r)^{\beta}$ is increasing with respect to $r \in[0,1)$. We only need to prove the following fact: for an increasing function $g:[0,1) \rightarrow[0, \infty), \alpha>0$ and $0<p \leq 1$,

$$
\left(\int_{0}^{1}(1-r)^{\alpha-1} g(r) d r\right)^{p} \leq C \int_{0}^{1}(1-r)^{p \alpha-1} g(r)^{p} d r .
$$

In fact, let $r_{k}=1-2^{-k}$. Using the monotonicity of $g$ and since $0<p \leq 1$, we have

$$
\begin{aligned}
& \left(\int_{0}^{1}(1-r)^{\alpha-1} g(r) d r\right)^{p} \\
& \quad=\left(\sum_{k=1}^{\infty} \int_{r_{k-1}}^{r_{k}}(1-r)^{\alpha-1} g(r) d r\right)^{p} \leq\left(\sum_{k=1}^{\infty} \int_{r_{k-1}}^{r_{k}} 2^{k}\left(1-r_{k-1}\right)^{\alpha} g\left(r_{k}\right) d r\right)^{p} \\
& \quad=\left(\sum_{k=1}^{\infty}\left(1-r_{k-1}\right)^{\alpha} g\left(r_{k}\right)\right)^{p} \leq \sum_{k=1}^{\infty}\left(1-r_{k-1}\right)^{p \alpha} g\left(r_{k}\right)^{p} \\
& \quad \leq C \sum_{k=1}^{\infty}\left(1-r_{k+1}\right)^{p \alpha} g\left(r_{k}\right)^{p} \leq C \sum_{k=0}^{\infty} \int_{r_{k}}^{r_{k+1}}(1-r)^{p \alpha-1} g(r)^{p} d r \\
& \quad=C \int_{0}^{1}(1-r)^{p \alpha-1} g(r)^{p} d r .
\end{aligned}
$$

This proves Lemma 2.1 .

LEMMA 2.2 ([6, Lemma 6]). For $0 \leq \rho<1$, and $\beta>\alpha>0$,

$$
\int_{0}^{1} \frac{(1-r)^{\alpha-1}}{(1-r \rho)^{\beta}} d r \leq \frac{C}{(1-\rho)^{\beta-\alpha}} .
$$

LEMMA 2.3. Let $\varphi$ be a normal function with characteristic exponents $a_{\varphi}$ and $b_{\varphi}$. For $p>0,0 \leq \rho<1$, if $s+t>b_{\varphi}$ and $s<a_{\varphi}$, then

$$
\int_{0}^{1} \frac{\varphi^{p}(r)}{(1-r)^{p s+1}(1-r \rho)^{p t}} d r \leq C \frac{\varphi^{p}(\rho)}{(1-\rho)^{p(s+t)}} .
$$


Using definitions of $a_{\varphi}$ and $b_{\varphi}$, the proof of Lemma 2.3 follows that of [4, Lemma 2.3].

Lemma 2.4 ([1]). Let $0<p \leq q \leq \infty, 0<\alpha, \beta<\infty$ and $\alpha+1 / p=\beta+1 / q$. Then for any measurable function $f$ on $B$

$$
\left(\int_{0}^{1}(1-r)^{q \beta-1} M_{q}^{q}(r, f) d r\right)^{1 / q} \leq C\left(\int_{0}^{1}(1-r)^{p \alpha-1} M_{p}^{p}(r, f) d r\right)^{1 / p} .
$$

LEMMA 2.5. Let $0<q<1$ and $s+t>n(1 / q-1)$. Then for any measurable function $f$ on $B$

$$
M_{q}\left(\rho, P_{s, t} f\right) \leq C(1-\rho)^{s}\left(\int_{0}^{1} \frac{r^{q(2 n-1)}(1-r)^{q(1+1)-2}}{(1-r \rho)^{q(n+s+r)-n}} M_{q}^{q}(r, f) d r\right)^{1 / q} .
$$

PROOF. Let

$$
F(z)=\frac{z^{2 n-1} f(z)}{(1-\langle z, w\rangle)^{n+s+t}}, \quad z=r \xi \quad \text { and } \quad w=\rho \zeta,
$$

where $\xi, \zeta \in \partial B$. Applying Lemma 2.4, equation (1.1) gives

$$
\begin{aligned}
\left|P_{s, r} f(w)\right|^{q} & \leq C(1-\rho)^{s q}\left(\int_{0}^{1}(1-r)^{t-1} \int_{\partial B}|F(r \xi)| d \sigma(\xi) d r\right)^{q} \\
& =C(1-\rho)^{s q}\left(\int_{0}^{1}(1-r)^{t-1} M_{1}(r, F) d r\right)^{q} \\
& \leq C(1-\rho)^{s q} \int_{0}^{1}(1-r)^{q(t+1)-2} M_{q}^{q}(r, F) d r \\
& =C(1-\rho)^{s q} \int_{0}^{1} \int_{\partial B} \frac{r^{q(2 n-1)}(1-r)^{q(t+1)-2}|f(r \xi)|^{q}}{|1-\langle r \xi, \rho \zeta\rangle|^{(n+s+t) q}} d \sigma(\xi) d r .
\end{aligned}
$$

Integrating on $\partial B$ with respect to $\zeta$, together with the formula in [5, Section 1.4.10], yield

$$
\begin{aligned}
M_{q}^{q}\left(\rho, P_{s, r} f\right) \leq & C(1-\rho)^{s q} \int_{0}^{1} r^{q(2 n-1)}(1-r)^{g(r+1)-2} \\
& \times \int_{\partial B}|f(r \xi)|^{q} \int_{\partial B} \frac{1}{\mid 1-\left\langle r \xi,\left.\rho \zeta\right|^{(n+s+i) q}\right.} d \sigma(\zeta) d \sigma(\xi) d r \\
\leq & C(1-\rho)^{s q} \int_{0}^{1} \frac{r^{q(2 n-1)}(1-r)^{q(t+1)-2}}{(1-r \rho)^{q(n+s+t)-n}} M_{q}^{q}(r, f) d r .
\end{aligned}
$$

Lemma 2.5 is proved. 
PROOF OF THEOREM 1.1. Let $f \in L^{p, q}(\varphi)$ and $g(z):=z^{2 n-1} f(z)$.

Case 1. $0<q<1, p \leq q$. Applying Lemmas 2.1,2.3 and 2.5 and the assumptions that $t>n(1 / q-1)+b_{\varphi}, s>-a_{\varphi}$, we have

$$
\begin{aligned}
\left\|P_{s, t} f\right\|_{p, q, \varphi}^{p} \leq & C \int_{0}^{1} \rho^{2 n-1}(1-\rho)^{s p-1} \varphi^{p}(\rho) \\
& \times\left(\int_{0}^{1} \frac{r^{q(2 n-1)}(1-r)^{q(t+1)-2}}{(1-r \rho)^{q(n+s+t)-n}} M_{q}^{q}(r, f) d r\right)^{p / q} d \rho \\
\leq & C \int_{0}^{1}(1-\rho)^{s p-1} \varphi^{p}(\rho)\left(\int_{0}^{1} \frac{(1-r)^{q(t+1)-2}}{(1-r \rho)^{q(n+s+t)-n}} M_{q}^{q}(r, g) d r\right)^{p / q} d \rho \\
\leq & C \int_{0}^{1}(1-\rho)^{s p-1} \varphi^{p}(\rho)\left(\int_{0}^{1} \frac{(1-r)^{p(t+1)-p / q-1}}{(1-r \rho)^{p(n+s+t)-n p / q}} M_{q}^{p}(r, g) d r\right) d \rho \\
= & C \int_{0}^{1} r^{p(2 n-1)}(1-r)^{p(t+1)-p / q-1} M_{q}^{p}(r, f) \\
& \times\left(\int_{0}^{1} \frac{(1-\rho)^{s p-1} \varphi^{p}(\rho)}{(1-r \rho)^{p(n+s+t)-n p / q}} d \rho\right) d r \\
\leq & C \int_{0}^{1} r^{p(2 n-1)}(1-r)^{p(1-n)(1-1 / q)-1} \varphi^{p}(r) M_{q}^{p}(r, f) d r \\
\leq & C \int_{0}^{1} r^{2 n-1}(1-r)^{-1} \varphi^{p}(r) M_{q}^{p}(r, f) d r=C\|f\|_{p, q, \varphi}^{p},
\end{aligned}
$$

where we used the change of variables $r^{p}=\rho$ and the inequality $\varphi^{p}\left(r^{1 / p}\right) \leq C \varphi(r)$. In fact, since $\varphi$ is normal, there exists $b>0$ and $0 \leq r_{0}<1$ such that $\varphi(r) /(1-r)^{b}$ is nondecreasing for $r_{0} \leq r<1$. So $r^{1 / p} \leq r$ implies that

$$
\varphi\left(r^{1 / p}\right) \leq \frac{\left(1-r^{1 / p}\right)^{b}}{(1-r)^{b}} \varphi(r) \leq C \varphi(r) .
$$

Case 2. $0<q<1, q<p<\infty$. Let $Q:=p / q$ and $1 / Q^{\prime}+1 / Q=1$. We select positive numbers $b_{1}, b_{2}, b_{3}$ and $b_{4}$ such that

(1) $0<q(t+1)-1=b_{1}+b_{2}=b_{3}+b_{4}$;

(2) $b_{3}>b_{1}$;

(3) $b_{2} / q+(n-1)(1-1 / q)>b_{\varphi}$;

(4) $a_{\varphi}>\left(b_{3}-b_{1}\right) / q-s$.

For example, for a sufficiently small number $\varepsilon>0$, we may take

$$
\begin{aligned}
& b_{1}=q(t+1)-1-(1+\varepsilon)\left(b_{\varphi}+(1-n)(1-1 / q)\right) q, \\
& b_{2}=(1+\varepsilon)\left(b_{\varphi}+(1-n)(1-1 / q)\right) q, \\
& b_{3}=q(t+1)-1-(1+\varepsilon)\left(b_{\varphi}+(1-n)(1-1 / q)\right) q+\varepsilon q,
\end{aligned}
$$


and

$$
b_{4}=(1+\varepsilon)\left(b_{\varphi}+(1-n)(1-1 / q)\right) q-\varepsilon q .
$$

By Lemmas 2.2, 2.3 and 2.5 and Hölder's inequality, we get

$$
\begin{aligned}
\left\|P_{s, t} f\right\|_{p, q, \varphi}^{p} \leq & C \int_{0}^{1} \rho^{2 n-1}(1-\rho)^{s p-1} \varphi^{p}(\rho) \\
& \times\left(\int_{0}^{1} \frac{(1-r)^{q(t+1)-2}}{(1-r \rho)^{q(n+s+1)-n}} M_{q}^{q}(r, g) d r\right)^{p / q} d \rho \\
\leq & C \int_{0}^{1}(1-\rho)^{s p-1} \varphi^{p}(\rho)\left(\int_{0}^{1} \frac{(1-r)^{Q^{\prime} b_{1}-1}}{(1-r \rho)^{Q^{\prime} b_{3}}} d r\right)^{Q / Q^{\prime}} \\
& \times \int_{0}^{1} \frac{(1-r)^{Q b_{1}-1}}{(1-r \rho)^{b^{\left(b_{4}-n+1+q(n+s-1) Q Q\right.}} M_{q}^{p}(r, g) d r d \rho} \\
\leq & C \int_{0}^{1}(1-\rho)^{s p+Q\left(b_{1}-b_{3}\right)-1} \varphi^{p}(\rho) \\
& \times \int_{0}^{1} \frac{(1-r)^{Q b_{2}-1}}{(1-r \rho)^{\left(b_{4}-n+1+q(n+s-1) Q\right.}} M_{q}^{p}(r, g) d r d \rho \\
= & C \int_{0}^{1} r^{p(2 n-1)}(1-r)^{Q b_{2}-1} M_{q}^{p}(r, f) \\
& \times \int_{0}^{1} \frac{(1-\rho)^{p\left(s+\left(b_{1}-b_{3}\right) / q\right)-1} \varphi^{p}(\rho)}{(1-r \rho)^{p\left(\left(b_{4}-n+1\right) / q+n+s-1\right)}} d \rho d r \\
\leq & C \int_{0}^{1} r^{p(2 n-1)}(1-r)^{p(1-n)(1-1 / q)-1} \varphi^{p}(r) M_{q}^{p}(r, f) d r \leq C\|f\|_{p, q, \varphi}^{p} .
\end{aligned}
$$

Case 3. $0<q<1, p=\infty$. Since $t>n(1 / q-1)+b_{\varphi}, s>-a_{\varphi}$, there exists $\beta>0$ such that $(n-1)(1-1 / q)+\beta+s>b_{\varphi}$ and $a_{\varphi}>\beta-t-1+1 / q$. In fact, from the definitions of $a_{\varphi}$ and $b_{\varphi}$, there exist $0<a_{0}<b_{0}$ and $0 \leq r_{0}<1$ such that $t>n(1 / q-1)+b_{0}, s>-a_{0}$, and $\varphi(r) /(1-r)^{a_{0}}$ is nonincreasing for $r_{0} \leq r<1$ with $\lim _{r \rightarrow 1^{-}}\left(\varphi(r) /(1-r)^{a_{0}}\right)=0, \varphi(r) /(1-r)^{b_{0}}$ is nondecreasing for $r_{0} \leq r<1$ with $\lim _{r \rightarrow 1^{-}}\left(\varphi(r) /(1-r)^{b_{0}}\right)=\infty$. Taking $\beta=(1-n)(1-1 / q)+a_{0}+b_{0}$. It is easy to check that $\beta$ satisfies the requirement.

Let $\psi(r)=(1-r)^{\beta} / \varphi(r)$,

$$
a^{\prime}=(1-n)\left(1-\frac{1}{q}\right)+a_{0} \quad \text { and } \quad b^{\prime}=(1-\bar{n})\left(1-\frac{1}{q}\right)+b_{0} .
$$

Then $\psi(r) /(1-r)^{a^{\prime}}=(1-r)^{b_{0}} / \varphi(r)$ is nonincreasing for $r_{0} \leq r<1$ and

$$
\lim _{r \rightarrow 1^{-}} \frac{\psi(r)}{(1-r)^{a^{\prime}}}=0
$$


$\psi(r) /(1-r)^{b^{\prime}}=(1-r)^{a_{0}} / \varphi(r)$ is nondecreasing for $r_{0} \leq r<1$ and

$$
\lim _{r \rightarrow 1^{-}} \frac{\psi(r)}{(1-r)^{b^{\prime}}}=\infty .
$$

Therefore $\psi(r)$ is a normal function.

From Lemmas 2.3 and 2.5, we obtain

$$
\begin{aligned}
\left\|P_{s, t} f\right\|_{\infty, q, \varphi} \leq & C \sup _{0 \leq \rho<1} \varphi(\rho)(1-\rho)^{s}\left(\int_{0}^{1} \frac{r^{q(2 n-1)}(1-r)^{q(t+1)-2}}{(1-r \rho)^{q(n+s+1)-n}} M_{q}^{q}(r, f) d r\right)^{1 / q} \\
\leq & C \sup _{0 \leq \rho<1} \varphi(\rho)(1-\rho)^{s} \\
& \times\left(\int_{0}^{1} \frac{(1-r)^{q(t+1)-2-\beta q} \psi(r)^{q}}{(1-r \rho)^{q(n+s+t)-n}} \varphi(r)^{q} M_{q}^{q}(r, f) d r\right)^{1 / q} \\
\leq & C\|f\|_{\infty, q, \varphi} \sup _{0 \leq \rho<1} \varphi(\rho)(1-\rho)^{s}\left(\int_{0}^{1} \frac{(1-r)^{q(t+1)-2-\beta q} \psi(r)^{q}}{(1-r \rho)^{q(n+s+t)-n}} d r\right)^{1 / q} \\
\leq & C\|f\|_{\infty, q, \varphi} \sup _{0 \leq \rho<1} \varphi(\rho) \psi(\rho)(1-\rho)^{(1-n)(1-1 / q)-\beta} \\
= & C\|f\|_{\infty, q, \varphi} \sup _{0 \leq \rho<1}(1-\rho)^{(1-n)(1-1 / q)} \leq C\|f\|_{\infty, q, \varphi} .
\end{aligned}
$$

This completes the proof of Theorem 1.1.

Finally we finish this note by stating a result on an application of Theorem 1.1 to Gleason's problem. Define $H^{p, q}(\varphi)$ to be the space of holomorphic functions on $B$ belonging to $L^{p, q}(\varphi)$. Gleason's problem on $H^{p, q}(\varphi)$ has been solved for the case $1 \leq q<\infty, 0<p<\infty$ (see, for example, [3] and [4]). The only unsolved case is $0<q<1,0<p<\infty$. As an application of Theorem 1.1, we solve Gleason's problem on $H^{p, q}(\varphi)$ for $0<q<1$ and $0<p \leq \infty$.

THEOREM 2.6. Gleason's problem can be solved on $H^{p, q}(\varphi)$ for $0<q<1$ and $0<p \leq \infty$. Precisely, for any integer $m>1$, there exist bounded linear operators $A_{\alpha}$ on $H^{p . q}(\varphi)$ such that if $f \in H^{p, q}(\varphi)$ and $D^{\alpha} f(0)=0(|\alpha| \leq m-1)$, then $f(z)=\sum_{|\alpha|=m} z^{\alpha} A_{\alpha} f(z)$ on $B$, where $D^{\alpha} f$ denotes the fractional derivative of $f$ of order $\alpha$, for $\alpha=\left(\alpha_{1}, \ldots, \alpha_{n}\right),|\alpha|=\left|\alpha_{1}\right|+\cdots+\left|\alpha_{n}\right|$.

The proof of Theorem 2.6 is similar to that of Theorem B in [3] and so is omitted.

\section{Acknowledgements}

The author would like to thank the referee for many useful suggestions and comments that made this paper a lot more readable. The paper was finished when the 
author visited the Center for Mathematics and its Applications (CMA) of Mathematical Sciences Institute at the Australian National University in 2003. The author thanks Professor Alan McIntosh for supporting his visit and CMA for their hospitality during the visit.

\section{References}

[1] F. Beatrous and J. Burbea, 'Holomorphic sobolev spaces on the ball', Dissertationes Math. (Rozprawy Mat.) 276 (1989), 60 pages.

[2] G. H. Hardy and J. E. Littlewood, 'Some properties of fractional integrals II', Math. Z. 34 (1932). 403-439.

[3] Y. Liu, 'Boundedness of the Bergman type operators on mixed norm spaces', Proc. Amer. Math. Soc. 130 (2002), 2363-2367.

[4] G. Ren and J. Shi, 'Bergman type operator on mixed norm spaces with applications', Chinese Ann. Math. Ser. B 18 (1997), 265-276.

[5] W. Rudin, Function theory in the unit ball of $\mathbb{C}^{n}$ (Springer, New York, 1980).

[6] A. L. Shields and D. L. Williams, 'Bounded projection, duality and multipliers in spaces of analytic functions', Trans. Amer. Math. Soc. 162 (1971), 287-302.

Department of Mathematics

Shantou University

Shantou Guangdong 515063

P. R. China

e-mail: zjlou@stu.edu.cn 